\title{
Partielle Lipodystrophie mit Verdacht auf Autoimmunerkrankung
}

Dargestellt wird der Fall eines 10-jährigen Jungen mit unklarer partieller Lipodystrophie im Bereich des Gesichtes, der im Rahmen des Innovationsfonds-Projektes TRANSLATE-NAMSE durch eine interdisziplinäre Fallkonferenz aufgeklärt werden konnte.

Das Versorgungsprojekt TRANSLATE-NAMSE strebt die bundesweite Verbesserung der medizinischen Versorgung von Patienten mit seltenen Erkrankungen an und wird aus dem Innovationsfonds des Gemeinsamen Bundesausschusses (G-BA) gefördert. Kern des Projektes bilden 9 universitäre Zentren für seltene Erkrankungen und 4 Institute für Humangenetik in Deutschland. Die gesetzlichen Krankenkassen AOK Nordost und BARMER sowie die Allianz Chronischer Seltener Erkrankungen e. V. (ACHSE) unterstützen das Vorhaben. Ein wichtiger Bestandteil des Projekts ist die Organisation interdisziplinärer, gegebenenfalls multizentrischer standortübergreifender Fallkonferenzen und eine gezielte Durchführung innovativer genetischer Diagnostik. So werden einzelne zentrale Maßnahmen des Nationalen Aktionsbündnis für Menschen mit seltenen Erkrankungen (NAMSE) Aktionsplans umgesetzt und eine beschleunigte präzise Diagnosestellung verfolgt (translate-namse.charite.de).

\section{Anamnese}

Im Alter von 7 Jahren fiel den Eltern bei dem Jungen erstmalig eine Verschmälerung im Bereich der Wangen auf. Hinzu kamen, wie die Familie berichtet, Haarausfall, rezidivierende Aphthen und Rückenschmerzen.

\section{Eigenanamnese}

Zustand nach Chemoprophylaxe mit Isoniazid für 3 Monate im Juli 2010 bei offener Tuberkulose (TBC) der Mutter ohne Anhalt für eine TBC-Infektion des Kindes (QuantiFERON ${ }^{\circledR}$ Tb-Gold Plus (IGRA) negativ, Röntgen-Thorax unauffällig).

\section{Erweiterte Anamnese}

Die Eltern und die 2 jüngeren Geschwister sind gesund.

- Tab. 1 Komplementanalytik im weiteren Verlauf (Universitätsklinikum Heidelberg, Institut für Immunlogie, Labor für Komplementdiagnostik, Dr. med. Jutta Schröder-Braunstein).

\begin{tabular}{|l|l|l|l|}
\hline Untersuchung & Ergebnis & Einheit & Referenzbereich \\
\hline Gesamtkomplement Klass. Weg/Funktion/1-Punkt & 3 & $\%$ & $65-135$ \\
\hline Gesamtkomplement Alternativweg/Funktion & 7 & $\%$ & $60-140$ \\
\hline Komplement C3d/Protein & 152 & $\mathrm{mU} / \mathrm{l}$ & $<40$ \\
\hline sC5b-9/terminaler Komplementkomplex & 634 & $\mathrm{ng} / \mathrm{ml}$ & $58-238$ \\
\hline C3-Nephritis-Faktor (C3NeF) & positiv & & negativ \\
\hline
\end{tabular}

\section{Untersuchungsbefund}

10-jähriger, altersgerecht entwickelter Junge in gutem Allgemeinzustand. Bis auf Lipodystrophie im Bereich der Wangen keine Auffälligkeiten. Gewicht und Größe sind altersgemäß.

\section{Laborbefunde}

Im Blutbild zeigten sich keine relevanten Veränderungen. Es fanden sich keine erhöhten Entzündungsparameter (negatives CRP und unauffällige BSG). Auffällig war eine Transaminasenerhöhung: GOT 55 U/I (17-50) und GPT $103 \mathrm{U} / \mathrm{I}$ (<41). Alkalische Phosphatase und LDH waren im Normbereich. Der direkte Coombs-Test war mit Bindung von anti-C3d positiv. Das 25-OH-Vitamin $\mathrm{D}_{3}$ war mit $19,8 \mathrm{nmol} / \mathrm{l}$ (50-150 nmol/l) erniedrigt.

In der Autoimmundiagnostik zeigte sich ein leicht erhöhter ANA-Titer mit 1:320 und ein positiver ENA-Screen. In der ENA-Differenzierung fanden sich positive anti-SS-A-/ Ro-Ak und anti-C1q-Antikörper. Der Nachweis des HLA-B27-Antigens war positiv.

Die Proteindiagnostik zeigte ein leicht erhöhtes Immunglobulin G mit 18,63 g/l (6,98-15,60), ein grenzwertig erhöhtes Immunglobulin A 2,06 g/I $(0,53-2,04)$ sowie ein deutlich erniedrigtes C3-Komplement mit 50 mg/l (900-1800). Immunglobulin $\mathrm{M}$, Ferritin und C4-Komplement waren im Normbereich.

Die weiterführende Komplementdiagnostik zeigte eine hochgradige Komplementaktivierung mit stark erniedrigtem C3 und deutlich erhöhten Aktivierungsprodukten
(C3d, sC5b-9) sowie eine Reduktion der Funktion des klassischen Wegs auf $5 \%$ (Referenzbereich 65-135\%) und des Alternativweges auf $10 \%$ (Referenzbereich 60 $140 \%)$. Der C3 Nephritis-Faktor (C3NeF) war positiv ( $\vee$ Tab. 1).

Im Spontanurin zeigte sich ein leicht erhöhtes Protein von 256,4 mg/dl (<150), das Kreatinin und der Kreatinin-Protein-Quotient lagen im Normbereich.

\section{Bildgebung und Lungen- funktionsuntersuchungen}

Die durchgeführte Magnetresonanztomografie des Kopfes, die Abdomensonografie und die Röntgenaufnahme des Thorax in 2 Ebenen zeigten Normalbefunde. Die Spirometrie- und Bodyplethysmografie waren ebenfalls ohne pathologischen Befund.

\section{Vorläufige Diagnose und Verlauf}

Aufgrund der Laborveränderungen mit erniedrigtem C3, positiven Anti-SSA/Ro-Ak, rezidivierenden Aphthen und Haarausfall bestand initial der Verdacht auf einen systemischen Lupus erythematodes mit Lipodystrophie. Es wurde eine immunsuppressive Therapie mit initialer oraler Glukokortikoidtherapie in Kombination mit Azathioprin und Hydroxychloroquin begonnen. Unter der Therapie kam es zu einer Normalisierung der Leberenzyme, die Lipodystrophie und die pathologischen Laborwerte ließen sich durch die Therapie allerdings nicht positiv beeinflussen. 
Die im Verlauf durchgeführte Komplementuntersuchung zeigte einen deutlich positiven C3NeF. Die Komplementanalysen ergaben keinen Hinweis auf einen homozygoten Komplementdefekt.

\section{Diagnose}

Aufgrund der zunächst unklaren Diagnose wurde der Patient in das oben beschriebene Versorgungsprojekt TRANSLATE-NAMSE eingeschlossen. Der Fall wurde unter Einschluss von Experten aus den Fachbereichen pädiatrische Rheumatologie, Immunologie, Nephrologie, Endokrinologie, Psychologie und Humangenetik diskutiert. Es konnte die Diagnose einer erworbenen partiellen Lipodystrophie (EPL) bzw. Barraquer-Simons-Syndrom (OMIM: 608709) gestellt werden. Die eingeleitete whole-exome-Sequenzierung erbrachte keine zugrundeliegende pathologische Mutation.

\section{Diskussion}

Das Krankheitsbild der EPL ist durch einen Verlust des subkutanen Fettgewebes, initi-al im Bereich des Gesichtes, gekennzeichnet. Typische laborchemische Merkmale sind sowohl eine Aktivierung des Komplementsystems (C3-Komplement-Erniedrigung bei normalem $C 4$ ), ein positiver C3 nephritic factor (C3NeF) als auch eine Erhöhung der oben beschriebenen Autoimmunparameter. Die Erkrankung zeigt eine Assoziation zum systemischen Lupus erythematodes sowie zur Dermatomyositis passend zu den positiven SSA-/ Ro-Antikörpern. Insgesamt wurden bisher mehr als 250 Fälle einer EPL beschrieben. Die Prävalenz wird auf 1-9/100 000 geschätzt (orphan.net).

In einem Literaturreview wurden Fallberichte von insgesamt 220 Patienten mit EPL aufgeführt, außerdem wurden 35 Patienten über einen Zeitraum von 8 Jahren beobachtet ( 1 body fat distribution, and prevalence of metabolic abnormalities in 35 patients with acquired partial lipodystrophy [APL]). Der durchschnittliche Erkrankungsbeginn lag in diesem Patientenkollektiv im 7. Lebensjahr. Im Verlauf kam es bei den Patienten zu einem weiteren bilateralen, symmetrischen Verlust des Fettgewebes, beginnend im Bereich des Gesichtes, Nackens, der oberen Extremität, Thorax und des Abdomens. Bei den beschriebenen Patienten bestand eine erhöhte Prävalenz für einen
Diabetes mellitus (6,7\%) sowie eine gestörte Glukosetoleranz (8,9\%). 83 \% der Patienten hatten ein erniedrigtes $\mathrm{C} 3$ und einen positiven C3NeF. Durchschnittlich 8 Jahre nach Beginn der Lipodystrophie entwickelten $22 \%$ der Patienten eine membranoproliferative Glomerulonephritis (MPGN). Die Patienten mit renaler Beteiligung waren signifikant früher erkrankt.

Seit langer Zeit ist bekannt, dass eine Dysregulation des Komplementsystems mit einer Nierenbeteiligung einhergehen kann. So wird bei einem hohen Anteil der Patienten mit einer C3-Glomerulonephritis ein positiver C3 nephritic factor (C3NeF) beobachtet. In manchen Fällen scheint die Anwesenheit dieses Autoantikörpers auch eine EPL zu verursachen. Der Zusammenhang zwischen Komplementsystem und Lipodystrophie wird detailliert von Corvillo et. al erläutert. Adipozyten sezernieren Komponenten des Komplementsystems und generieren das Enzym C3-Konvertase (C3b$3 \mathrm{Bb}$ ). Ein vorliegender $\mathrm{C} 3 \mathrm{NeF}$ stabilisiert diese C3-Konvertase und führt so zu einer unkontrollierten Aktivierung des alternativen Weges in den Zellmembranen. Dieser 
Prozess führt dann im Verlauf zur Lyse der Adipozyten [2].

Lediglich bei einer kleinen Zahl an Patienten mit einer erworbenen partiellen Lipodystrophie wurden krankheitsassoziierte Mutationen im Lamin B2 (LMNB2)-Gen beschrieben [3, 4]. Es gibt keine weiteren Hinweise darauf, dass genetische Defekte mit einer erworbenen partiellen Lipodystrophie in Verbindung gebracht werden können.

Die Prognose der EPL hängt weitestgehend von dem Ausmaß der Nierenbeteiligung ab. Unserem Patienten wurden regelmäßige Urinkontrollen empfohlen sowie eine Umstellung der Therapie von Azathioprin auf Mycophenolat-Mofetil. Sollte es im Verlauf zu einer Nierenbeteiligung kommen, kann eine Inhibition der terminalen Komplementaktivierung mit dem monoklonalen anti-C5-Antikörper Eculizumab erwogen werden [5]. Zur Therapie möglicher metabolischer Anomalien stände gegebenenfalls Metreleptin zur Verfügung.

Interessenkonflikt

Die korrespondierende Autorin erklärt, dass in Bezug auf diese Arbeit für keinen der Autorinnen und Autoren ein Interessenkonflikt besteht.
A. Raab', M. Danyel'2, R. Thalemann', D. Müller ${ }^{3}$, D. Horn², H. Krude ${ }^{4}$, J. Schröder-Braunstein ${ }^{5}$, T. Kallinich ${ }^{1}$

'Universitätskinderklinik Charité, Campus Virchow, Klinik für Pädiatrie m. S. Pneumologie, Immunologie und Intensivmedizin, SPZ, Sektion Pädiatrische Rheumatologie

IInstitut für Medizinische Genetik und Humangenetik, Charité, Berlin ${ }^{3}$ Klinik für Pädiatrie mit Schwerpunkt Gastroenterologie, Nephrologie und Stoffwechselmedizin, Charité, Berlin ${ }^{4}$ Institut für Experimentelle Pädiatrische Endokrinologie, Charité, Berlin 5 Institut für Immunologie, Universitätsklinikum Heidelberg

\section{Korrespondenzadresse}

Dr. Anna Raab

Universitätsklinikum Charité, Campus Virchow

Klinik für Pädiatrie mit Schwerpunkt Pneumologie, Immunologie und Intensivmedizin

SPZ, Sektion Pädiatrische Rheumatologie Charitéplatz 1, 10117 Berlin anna.raab@charite.de
Literatur

[1] Misra A, Peethambaram A, Garg, A. Clinical Features and Metabolic and Autoimmune Derangements in Acquired Partial Lipodystrophy. Medicine 2004; 83(1): 18-34. https://doi.org/10.1097/01. md.0000111061.69212.59

[2] Corvillo F, López-Trascasa M. An overview of lipodystrophy and the role of the complement system. Molecular Immunology 2019; 112: 223-232. https://doi.org/10.1016/j. nefro.2017.10.002

[3] Gao J, Li Y, Fu X, Luo X. A Chinese patient with acquired partial lipodystrophy caused by a novel mutation with LMNB2 gene. Journal of Pediatric Endocrinology \& Metabolism 2012; 25(3-4): 375-377. Retrieved from http://www.ncbi.nlm.nih.gov/pub$\operatorname{med} / 22768673$

[4] Capeau J, Magré J, Caron-Debrale M et al. $\mathrm{t}$ al. Human lipodystropies: genetic and acquired diseases of adipose tissue. Endocr Dev 2010; 19: 1-20

[5] Ozkaya O, Nalcacioglu H, Tekcan D et al. Eculizumab therapy in a patient with dense-deposit disease associated with partial lipodystropy. Pediatric Nephrology 2014; 29(7): 1283-1287. https://doi. org/10.1007/s00467-013-2748-5

Bibliografie

DOI https://doi.org/10.1055/a-1011-1319 arthritis + rheuma 2019; 39: 341-343 (c) Georg Thieme Verlag KG Stuttgart · New York ISSN 0176-5167 